\title{
Synergistic Contributions of Multi-Walled Carbon Nanotubes and Cobalt Nitroprusside Film toward Improved Performance for a Glucose Sensor
}

\author{
Xiao-Ping $\mathrm{HONG}^{1,}$ and Jing-Ying $\mathrm{MA}^{2, \mathrm{~b}}$ \\ ${ }^{1}$ Department of Chemistry, Zhejiang Sci-Tech University, Hangzhou 310018, China; \\ ${ }^{2}$ Department of Construction, Zhejiang College of Construction, Hangzhou 311231, China. \\ axpnghong@163.com, ${ }^{\mathrm{a}}$ majy75@163.com
}

Keywords: Carbon nanotubes, Cobalt nitroprusside, Pentacyanonitrosylferrate, Modified electrode, Glucose.

\begin{abstract}
The preparation and attractive performance of cobalt nitroprusside/multi-walled carbon nanotubes (CoNP/MWCNTs) composite electrodes, based on the electrochemical deposition of CoNP onto the surface of MWCNTs modified electrode, was described. The resulting CoNP/MWCNTs composites brought new capabilities for electrochemical devices by combining the advantages of MWCNTs and CoNP. The marked electrocatalytic activity of the new composite electrode permitted sensitive and low-potential amperometric determination of glucose. The consecutive cyclic voltammetry and energy dispersion X-ray spectroscopy (EDX) characterization demonstrated the successful fabrication of CoNP/MWCNTs composites on the surface of electrode. The comparison of voltammetric responses of three types of working electrodes (CoNP/MWCNTs composite electrode, MWCNTs modified electrode and glassy carbon electrode, respectively) toward glucose, as well as the interference of uric acid and ascorbic acid, was examined. The combination of the excellent properties of MWCNTs and fantastic electrocatalytic performance of CoNP allowed building up a robust, sensitive, selective, and stable amperometric biosensor toward glucose.
\end{abstract}

\section{Introduction}

Since the work of Iijima in 1991, carbon nanotubes (CNTs) initiated a worldwide explosion of research on its application. On the basis of its good electro catalytic activities, nanometer size effect and surface fouling resistance, CNTs and its functionalized forms attract a lot of research activity. Up to now, CNTs has been successfully used for hydrogen storage materials [1], CNT-based electronic components [2], CNT-based electrodes [3], sensors [4], filters and membranes [5], biomedical applications [6] and composite materials [7].

Based on the properties of huge specific surface area, perfect surface antifouling performance, excellent electron-transfer capability and fantastic electrochemical catalysis capacity [8], CNTs are very promising for application of electrochemical sensors or electrochemical modified electrodes. In the development of electrochemical sensors, the sensitivity and the selectivity are the crucial issues for their properties. Much effort has been made to improve the two properties by enhancing current response [9], reducing over potential [10] and inhibiting interferences [11]. Extensive study of using CNTs as electrodes materials proved that this kind of carbon allotrope imparts significant advantages of high current response, low work potential and electrode surface antifouling performance. Wang's group developed a CNT/Nafion electrode, which successfully enhanced the voltammetric response of dopamine (DA) and effectively suppressed the interference of uric acid (UA) and ascorbic acid (AA) [12]. Valentini et al. fabricated a hydroxyl group functionalized SWCNTs modified electrode for epinephrine (EP) determination [13]. Due to the excellent electro catalytic activities of multi-walled carbon nanotubes (MWCNTs), A MWCNTs and chitosan (CS) composite film modified electrode was also fabricated by layer-by-layer method for trace nictitate adenine dicucleotide (NADH) direct electrochemical determination at a relatively low potential (0.4 V) [14]. Based on the remarkable faster electron transfer capability of CNT, trace sulfadiazine residue was sensitively and selectively 
monitored in our previously reported work, in which the anodic potential of sulfadiazine was $250 \mathrm{mV}$ lower than that of normal glassy carbon (GC) electrode [15].

Metal pentacyanonitrosylferrate $\left(\mathrm{Fe}(\mathrm{CN})_{5} \mathrm{NO}^{2-}\right)$ is also referred as metal nitroprusside (MNP), which have received much attention of application as electrochemical redox mediators due to its versatility and electro catalytic acivity [16]. In pervious publications, MNP thin films or small particles were deposited onto the surface of normal electrodes using different strategies. Reducible analytes, such as ascorbic acid, hydrogen peroxide, sulfide, sulfite, etc. were successfully determined with MNP functionalized sensors.

In present work, an attempt has been made for the first time to combine cobalt nitroprusside (CoNP) to MWCNTs to form CoNP/MWCNTs composites. And a CoNP/MWCNT composite electrode was fabricated by electrochemical deposition method for the measurement of glucose.

\section{Materials and Methods}

A CHI 660A electrochemical workstation (CH Instruments Inc., Austin, USA) was used for electrochemical testing. The electrochemical cell was composed of an $\mathrm{Ag} / \mathrm{AgCl}$ (saturated $\mathrm{KCl}$ ) reference electrode, a Pt wire auxiliary electrode and a series of work electrodes (GC electrode, MWCNTs modified electrode, CoNP/MWCNT composite electrode, respectively). Indium-tin oxide (ITO) glass was selected as the substrate for energy dispersion X-ray spectroscopy (EDX) characterization.

MWCNTs crude material (diameter 10-30 nm, length 5-15 $\mu \mathrm{m}$, purity $>95 \%$ ) was obtained from Shenzhen Nanotech Port, China. And the crude MWCNTs were pretreated prior to use according to the strategy of Liu et al. [22]. Sodium nitroprusside (NaNP) and other analytical grade reagents were purchased from Micxy Reagent, China. All solutions were prepared with Milli-Q water from an Academic Millipore system (Millipore Inc., MA, USA).

The preparation of the CoNP/MWCNT composite electrode was carried out in three steps. Firstly, pretreated MWCNTs was dispersed into $1 \mathrm{~g} / \mathrm{L}$ sodium polystyrene sulfonate (PSS) solution with the help of sonication to form a $1 \mathrm{mg} / \mathrm{mL}$ of suspension. And a drop of above-mentioned suspension (2 $\mu \mathrm{L}$ ) was cast onto the surface of a cleaned GC electrode. After volatilizing the solvent, a MWCNTs modified electrode was obtained. Secondly, metallic Co was electrodeposited onto the MWCNTs modified electrode from $0.05 \mathrm{M} \mathrm{CoCl}_{2}$ solution by application of a constant cathodic potential of $-1.15 \mathrm{~V}$ for $400 \mathrm{~s}$. Finally, CoNP was electrochemically prepared and deposited onto the surface of MWCNTs electrode by consecutively cyclic voltammetric scaning (30 times) in electrolyte containing $0.05 \mathrm{M} \mathrm{NaNP}$ and $0.25 \mathrm{M} \mathrm{KNO}_{3}$ in the potential range 0.2 to $0.75 \mathrm{~V}$ at a scan rate of 50 $\mathrm{mV} \mathrm{s}^{-1}$. Another CoNP/MWCNT electrode with ITO glass substrate was also prepared with the same procedure mentioned above for the EDX characterization (EDX, Hitachi, Japan).

\section{Results and Discussion}

\section{Characterization of the CoNP/MWCNT Composite Electrode.}

The CoNP/MWCNT composite electrode was fabricated by consecutive cyclic voltammetric scanning in an electrolyte containing $0.05 \mathrm{M}$ NaNP and 0.25 M KNO3, where a MWCNTs modified electrode covered with Co acted as the working electrode. After 30 cycles, a stable and overlapping voltammogram was observed (Fig. 1A), which indicated the CoNP film growing onto the sidewalls of the MWCNTs gradually and finally leveling out. The typical redox peaks arised at about $0.52 \mathrm{~V}$ vs. $\mathrm{Ag} / \mathrm{AgCl}$ (saturated $\mathrm{KCl}$ ) is attributed to the following reaction(Eq. 1):

$$
\mathrm{Co}^{\mathrm{II}}\left[\mathrm{Fe}^{\mathrm{III}}(\mathrm{CN})_{5} \mathrm{NO}\right]+\mathrm{K}^{+}+\mathrm{e}^{-} \leftrightarrow \mathrm{KCo}^{\mathrm{II}}\left[\mathrm{Fe}^{\mathrm{II}}(\mathrm{CN})_{5} \mathrm{NO}\right]
$$

A CoNP/MWCNTs composite electrode based on ITO substrate was also fabricated for EDX analysis. As is shown in Fig. 1B, the dominating carbon (C) peak, attributed to the MWCNTs immobilized on the electrode surface, is expected. Strong peaks of silicon (Si) and indium (In) due to 
the ITO substrate are also observed. Well-marked EDX peaks of Co $(0.78,6.9,7.7 \mathrm{keV})$ and $\mathrm{Fe}(0.71$, $6.4 \mathrm{keV}$ ) are also obtained in the EDX spectrum, which confirms that the existence of Co and Fe elements on the surface of modified electrode. It coincides with the above conclusion of the formation of CoNP/MWCNTs composites during the consecutive cyclic voltammetric scanning (Fig. 1 A).
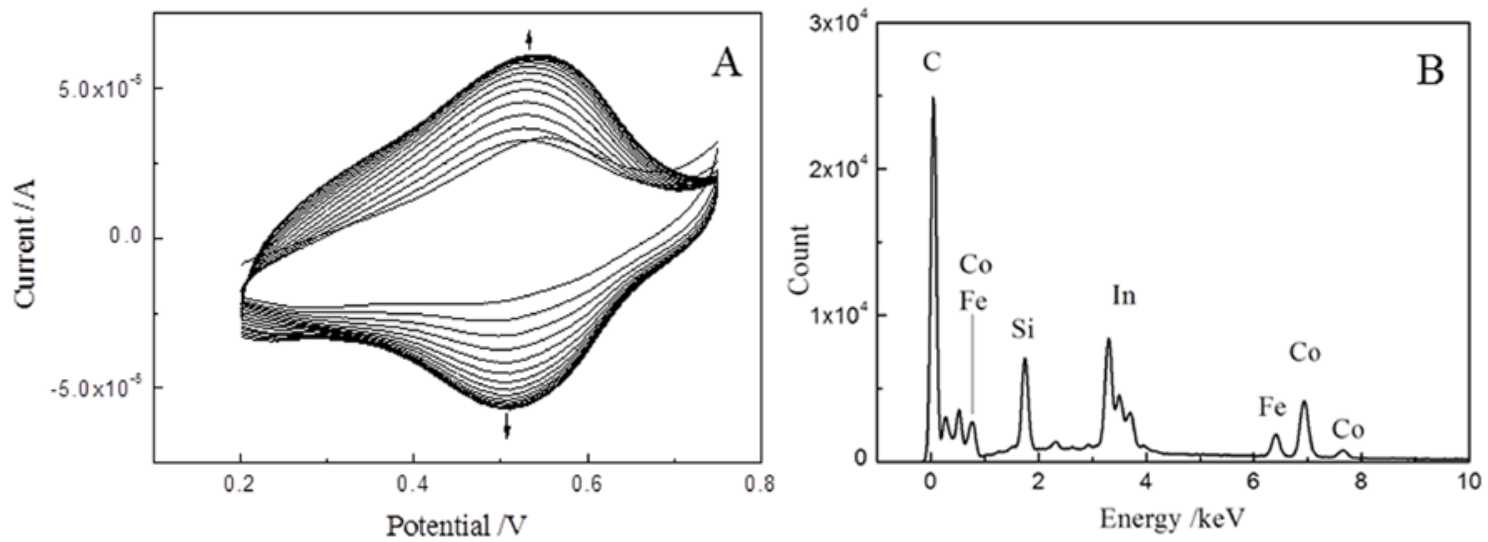

Fig. 1 Electrochemical fabrication of CoNP/MWCNTs composite electrode (A) and EDX spectrum of CoNP/MWCNTs composite electrode with ITO substrate (B)

\section{Electrocatalytic Performance of CoNP/MWCNTs Composite Electrode toward Glucose.}

Diabetes mellitus, metabolic disorder of glucose, is of a was major public health problem affecting about millions of people worldwide. In this section, glucose used as the probe for characterizing the electrocatalytic performance of CoNP/MWCNTs composite electrode. As shown in Fig. 2, cyclic voltammograms of CoNP/MWCNTs composite electrode, MWCNTs modified electrode and GC electrode were recorded separately in $0.1 \mathrm{M} \mathrm{NaOH}$ solution with the presence and absence of $1.0 \mathrm{mM}$ glucose at a scan rate of $50 \mathrm{mV} \mathrm{s}^{-1}$. With the presence of glucose, a negligible anodic current rose gradually at about $0.5 \mathrm{~V}$ was obtained on GC electrode (curve 4, Fig. 2) while a weak anodic current wave around 0.5 V was observed on MWCNTs modified electrode (curve 3, Fig. 2). Due to the high anodic potential and low response, both of GC electrode and MWCNTs modified electrode are unsuitable for direct electrochemical measurement of glucose. With the absence of glucose, the CoNP/MWCNTs composite electrode displayed a rather steady background response without any anodic wave (curve 2, Fig. 2). In contrast, a well-defined anodic current wave rose at about $0.3 \mathrm{~V}$ with the presence of glucose when the CoNP/MWCNTs composite electrode was used as the working electrode. Therefore, it can be concluded that the enhanced current and low anodic potential response observed on the CoNP/MWCNTs composite electrode were attribute to the synergistic effect of CoNP and MWCNTs coated on the electrode surface.

\section{Amperometric Sensing of CoNP/MWCNTs Composite Electrode toward Glucose.}

The amperometric response at CoNP/MWCNTs composite electrode in electrolyte of $0.1 \mathrm{M} \mathrm{NaOH}$ at an applied potential of $0.35 \mathrm{~V}$ vs. AgCl/Ag (saturated $\mathrm{KCl}$ ) was evaluated in Fig. 3 by dropwise successive addition of glucose in concentration of $0.01 \mathrm{M}$. The composite electrode yielded a remarkable current response toward glucose addition. For each addition of glucose $(0.01 \mathrm{M})$, the response current increased steeply to reach a stable value in less than $3 \mathrm{~s}$, confirming high sensitivity and fast response. A linear relationship of the current response against glucose concentration $(I(\mathrm{~A})=$ $0.00932 \times C(\mathrm{M})+1.093 \times 10^{-6}$ ) was obtained in the range $0.04-2.3 \mathrm{mM}$ with the correlation coefficient $R$ 0.997. The detection limit of $0.02 \mathrm{mM}$ was calculated according to signal/noise ratio of 3 . The above mentioned superior performance probably attribute to the high electrocatalytic activity of CoNP/MWCNTs composite electrode. 


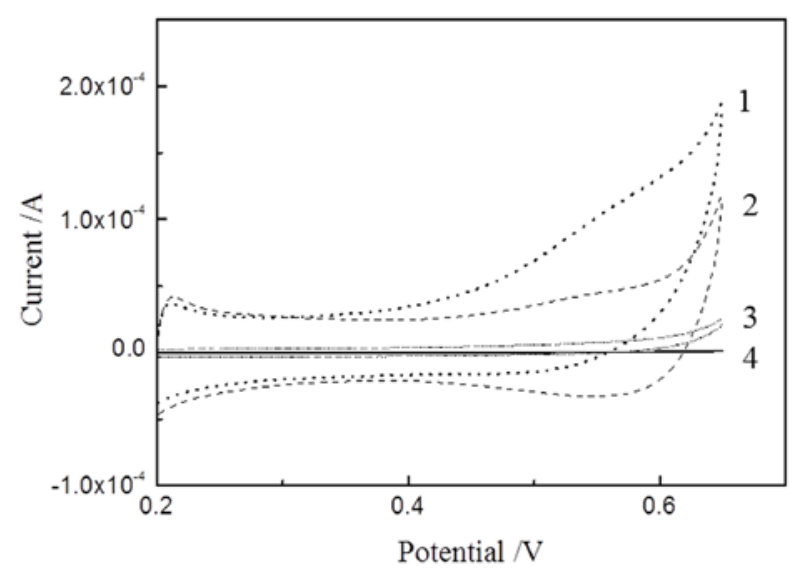

Fig. 2 Cyclic voltammetric response of CoNP/MWCNTs composite electrode (1, 2), MWCNTs modified electrode (3) GC electrode (4) in $0.1 \mathrm{M} \mathrm{NaOH}$ solution with the presence $(1,3,4)$ and absence (2) of $1.0 \mathrm{mM}$ glucose at a scan rate of $50 \mathrm{mV} \mathrm{s}^{-1}$

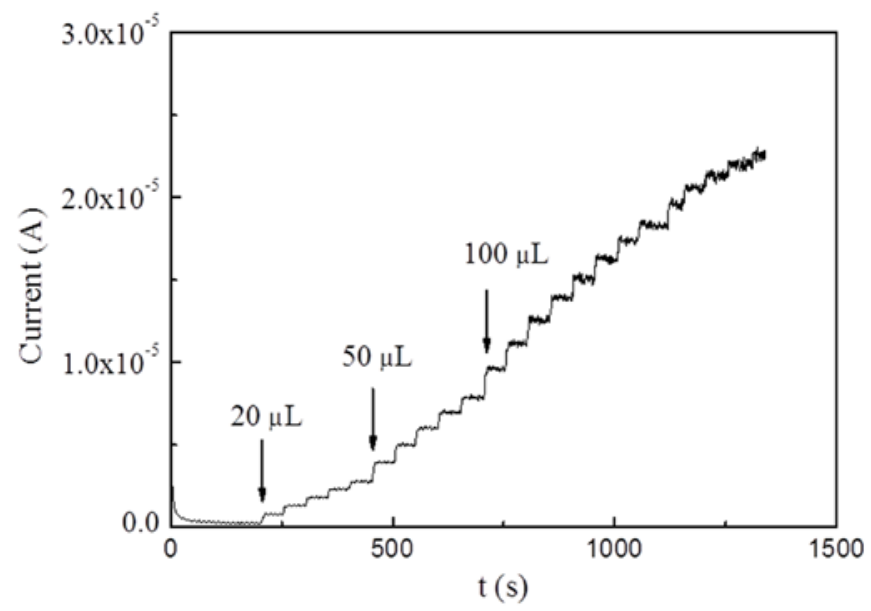

Fig. 3 Chronoamperometric response of CoNP/MWCNTs composite electrode toward successive addition of different volumn of $1.0 \mathrm{mM}$ glucose into $0.1 \mathrm{M} \mathrm{NaOH}$ electrolyte

\section{Interference Study.}

To eliminate the inference of many symbiotic compounds, a low work potential $0.35 \mathrm{~V}$ was applied in the whole amperometric procedure to avoid the oxidation of the majority interfering species. Due to the low anodic potential of uric acid (UA) and ascorbic acid (AA), the two interferences are probable the major challenges during amperometric determination of glucose. In light of the content of UA $(0.1 \mathrm{mM})$ and AA $(0.1 \mathrm{mM})$ in a normal physiological sample is much lower than that of glucose (3-8 $\mathrm{mM}$ ), the anodic current responses of UA and AA are very weak. With the addition of $0.1 \mathrm{mM} \mathrm{UA}$ (Fig. 4, a) and AA (Fig. 4, d) into $0.1 \mathrm{M} \mathrm{NaOH}$ solution, the current responses were almost negligible. However, remarkable signals were observed when $1.0 \mathrm{mM}$ glucose spiked into the electrolyte (Fig. 4, b, c). A reproducible current response $(\mathrm{RSD}=2.3 \%)$ was obtained at CoNP/MWCNTs composite electrode for 8 measurements of a standard glucose solution. The stability of CoNP/MWCNTs composite electrode was examined by storing the new modified electrode at ambient condition and checking the current response everyday at the same condition. And the current response of the composite electrode maintained $97.9 \%$ of its initial response after 15days. Hence, the results mentioned above indicate that the composite electrode is selective and stable for glucose measurement. 


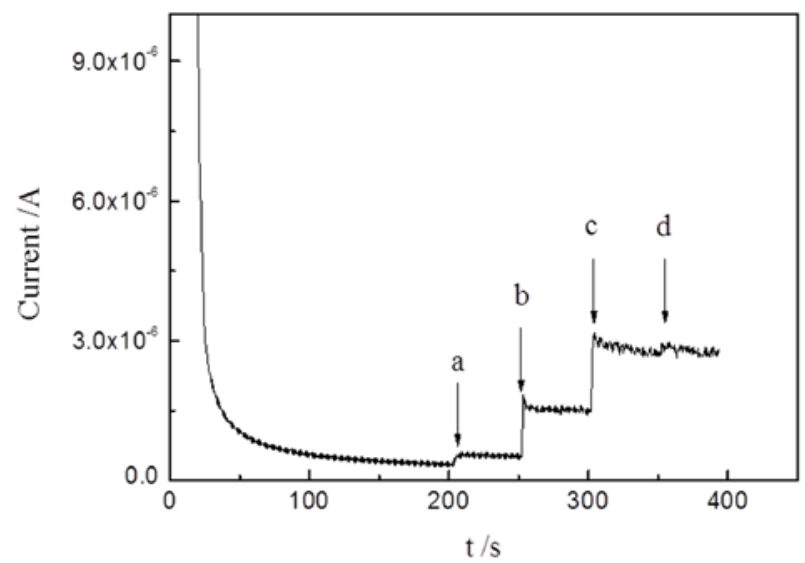

Fig. 4 Chronoamperometric response of CoNP/MWCNTs composite electrode to addition of $0.1 \mathrm{mM}$ UA (a), $1.0 \mathrm{mM}$ glucose (b and c) and $0.1 \mathrm{mM}$ AA (d) into $0.1 \mathrm{M} \mathrm{NaOH}$ electrolyte

\section{Conclusion}

In conclusion, a glucose biosensor based on CoNP/MWCNTs composite, was fabricated by electrochemical deposition of electrocatalytic components CoNP onto the surface of MWCNTs electrode. The as-prepared composite electrode exhibited electrochemical detection of glucose over a wide linear range $(0.04-2.3 \mathrm{mM})$ with an impressive detection limit $(0.01 \mathrm{mM})$ due to the synergistic effect of CoNP, MWCNTs and PSS.

\section{Acknowledgement}

This work was financially supported by the Scientific Research Fund of Science Technology Department of Zhejiang Province (Project No. 2015C37014) and the Science Foundation of Zhejiang Sci-Tech University (Project No. 1206819-Y)

\section{References}

[1] A. Nikitin, X.L. Li, Z.Y. Zhang, Hydrogen storage in carbon nanotubes through the formation of stable C-H bonds, Nano Lett. 8 (2008) 162-167.

[2] J. Oh, G. Yoo, Y.W. Chang, A carbon nanotube metal semiconductor field effect transistor-based biosensor for detection of amyloid-beta in human serum, Biosens. Bioelectron. 50 (2013) 345-350.

[3] Y.Y. Yu, Z.G. Chen, S.J. He, Direct electron transfer of glucose oxidase and biosensing for glucose based on PDDA-capped gold nanoparticle modified graphene/multi-walled carbon nanotubes electrode, Biosens. Bioelectron. 52 (2014) 147-152.

[4] D. Ragupathy, A.I. Gopalan, K.-P. Lee, Synergistic contributions of multiwall carbon nanotubes and gold nanoparticles in a chitosan-ionic liquid matrix towards improved performance for a glucose sensor, Electrochem. Commun. 11 (2009) 397-401.

[5] M. Kumar, M. Ulbricht, Novel antifouling positively charged hybrid ultrafiltration membranes for protein separation based on blends of carboxylated carbon nanotubes and aminated poly(arylene ether sulfone), J. Membrane Sci. 448 (2013) 62-73.

[6] W.R. Yang, P. Thordarson, J. Gooding, Carbon nanotubes for biological and biomedical applications, Nanotechnology 18 (2007) 1-12. 
[7] E. Dervishi, F. Hategekimana, L. Boyer, The effect of carbon nanotubes and graphene on the mechanical properties of multi-component polymeric composites, Chem. Phys.Lett.. 590 (2013) 126-130.

[8] B. Pérez-López, A. Merkoçi, Carbon nanotubes and graphene in analytical sciences, Microchim. Acta 179 (2012) 1-16.

[9] L. Zheng, J.F. Song, Ni(II)-baicalein complex modified multi-wall carbon nanotube paste electrode toward electrocatalytic oxidation of hydrazine, Talanta 79 (2009) 319-326.

[10] S. Shahrokhian, L. Fotouhi, Carbon paste electrode incorporating multi-walled carbon nanotube/cobalt salophen for sensitive voltammetric determination of tryptophan, Sensor. Actuat. B-Chem. 123 (2007) 942-949.

[11] S.R. Ali, Y.F. Ma, R.R. Parajuli, A nonoxidative sensor based on a self-doped polyaniline/carbon nanotube composite for sensitive and selective detection of the neurotransmitter dopamine, Anal. Chem. 79 (2007) 2583-2587.

[12] H.-S. Wang, T.-H. Li, W.-L. Jia, Highly selective and sensitive determination of dopamine using a Nafion/carbon nanotubes coated poly(3-methylthiophene) modified electrode, Biosens. Bioelectron. 22 (2006) 664-669.

[13] F. Valentini, G. Palleschi, E.L. Morales, Functionalized single-walled carbon nanotubes modified microsensors for the selective response of epinephrine in presence of ascorbic acid, Electroanal, 19 (2007) 859-869.

[14] X. Zhai, W. Wei, J. Zeng, Lay-by-layer assembled film based on chitosan/carbon nanotubes, and its application to electrocatalytic oxidation of NADH, Microchim. Acta 154 (2006) 315-320.

[15] X.-p. Hong, Y. Zhu, Y.-z. Zhang, Electrocatalytic response of poly(cobalt tetraaminophthalocyanine)/multi-walled carbon nanotubes-Nafion modified electrode toward sulfadiazine in urine, J. Zhejiang Univ.-Sc. B 13 (2012) 503-510.

[16] L.L. Paim, N.R. Stradiotto, Electrooxidation of sulfide by cobalt pentacyanonitrosylferrate film on glassy carbon electrode by cyclic voltammetry, Electrochim. Acta 55 (2010) 4144-4147. 